\title{
Expansion and diversification of the gibberellin receptor GIBBERELLIN INSENSITIVE DWARF1 (GID1) family in land plants
}

\author{
Rajesh K. Gazara, Kanhu C. Moharana, Daniel Bellieny-Rabelo" and Thiago M. Venancio*
} (1)

Laboratório de Química e Função de Proteínas e Peptídeos, Centro de Biociências e Biotecnologia, Universidade Estadual do Norte Fluminense Darcy Ribeiro; Campos dos Goytacazes, Brazil.

\# Present address: Department of Microbiology and Plant Pathology, University of Pretoria, Lunnon Road, Pretoria, South Africa, 0028.

\author{
* Corresponding author \\ Av. Alberto Lamego 2000 / P5 / 217; Parque Califórnia

\section{ABSTRACT}

Gibberellic acid (gibberellins, GA) controls key developmental processes in the life-cycle of land plants. By interacting with the GIBBERELLIN INSENSITIVE DWARF1 (GID1) receptor, GA regulates the expression of a wide range of genes through different pathways. Here we report the systematic identification and classification of GID1s in 52 plants genomes, encompassing from bryophytes and lycophytes, to several monocots and eudicots. We investigated the evolutionary relationship of GID1s using a comparative genomics framework and found strong support a previously proposed phylogenetic classification of this family in land plants. We identified lineage-specific expansions of particular subfamilies (i.e. GID1ac and GID1b) in different eudicot lineages (e.g. GID1b in legumes). Further, we found both, shared and divergent structural features between GID1ac and GID1b subgroups in eudicots that provide mechanistic 
31 insights on their functions. Gene expression data from several species show that at least one

32 GID1 gene is expressed in every sampled tissue, with a strong bias of GID1b expression towards underground tissues and dry legume seeds (typically with low GA levels). Taken together, our results indicate that GID1ac retained canonical GA signaling roles, whereas GID1b specialized in conditions of low GA concentrations. We propose that this functional specialization occurred initially at the gene expression level and was later fine-tuned by specific mutations that conferred greater GA affinity to GID1b, including a Phe residue in the GA-binding pocket. Finally, we discuss the importance of our findings to understand the diversification of GA perception mechanisms in land plants.

Keywords: GID1, gibberellin signaling, whole genome duplication, gene expression divergence.

\section{INTRODUCTION}

Gibberellins (GAs) are hormones that regulate various processes in plant development, particularly during seed germination, flowering, pollen development and stem elongation

46 (Olszewski et al. 2002). The classic GA signaling pathway is characterized by the recognition of

47 bioactive $\mathrm{GA}$ (e.g. $\mathrm{GA}_{3}$ and $\mathrm{GA}_{4}$ ) by the receptor GIBBERELLIN INSENSITIVE DWARF1

48 (GID1). GID1 is a nucleocytoplasmic protein (Livne and Weiss 2014) that was initially

49 identified in rice (OsGID1, Oryza sativa) (Ueguchi-Tanaka et al. 2005). Upon interaction with

50 GA, GID1 undergoes a conformational change that increases its affinity for DELLA, proteins

51 that typically inhibit GA signaling by: interacting and blocking the activity of transcription

52 factors that drive GA transcriptional programs (Murase et al. 2008); working as co-activators of 53 negative regulators of GA signaling and; recruiting chromatin remodeling proteins to promoter 
54 regions (Nelson and Steber 2016). In the canonical GA signaling pathway, the GA-GID155 DELLA complex is recognized by the $\mathrm{SCF}^{\mathrm{SLY} 1}$ ubiquitin ligase, which ubiquitinates DELLA 56 proteins, promoting their proteasomal degradation (Dill et al. 2004; Fu et al. 2004; Gomi et al. 57 2004; McGinnis et al. 2003; Peng et al. 1997). Therefore, the down-regulation of DELLA is the 58 process that ultimately trigger the classic GA effects (Fleet and Sun 2005). Alternative GA 59 signaling pathways have also been proposed, such as a GA-independent (GID1-mediated) (Yamamoto et al. 2010) and DELLA-independent pathways (Fuentes et al. 2012). Interestingly,

61 both the canonical and alternative pathways described above rely on GID1, which appears to 62 have a central role in GA signaling. Comparison of HSLs with the rice GID1 revealed important differences: the His from the HSL catalytic triad (Ser-Asp-His) is replaced by Val in GID1; the last Gly of the HGGG motif is substituted by Ser in GID1 and; the extensive divergence between N-terminal lid of GID1 and HSLs (Hirano et al. 2012). Detailed structural analyses of the GA-GID1a-DELLA complex support that these changes are critical for GA binding. Other GID1a amino acid residues were also found to be involved in GA interaction: Gly ${ }^{114}, \mathrm{Gly}^{115}, \mathrm{Ser}^{116}, \mathrm{Ile}^{126}, \mathrm{Tyr}^{127}, \mathrm{Ser}^{191}, \mathrm{Phe}^{238}$, $70 \operatorname{Val}^{239}, \mathrm{Asp}^{243}, \mathrm{Arg}^{244}, \mathrm{Tyr}^{247}, \mathrm{Gly}^{320}, \mathrm{Tyr}^{322}, \mathrm{Leu}^{323}$ (core domain residues) and; Ile ${ }^{24} \mathrm{Phe}^{27}$, $71 \mathrm{Lys}^{28}, \mathrm{Tyr}^{31}, \mathrm{Arg}^{35}$ (N-terminal extension residues) (Murase et al. 2008).

Three GID1 receptor genes have been characterized in Arabidopsis thaliana (GID1a, GID1b and GID1c). Although some level of functional redundancy was found between these

74 genes, each of them apparently play specific roles in different developmental stages (Griffiths et 
77 2008), barley (Chandler et al. 2008) and wheat ( $\mathrm{Li}$ et al. 2013). A previous phylogenetic reconstruction of GID1 receptors uncovered the presence of three major groups: eudicot GID1ac, eudicot GID1b and monocot GID1, supporting that a diversification of this family occurred after the split of monocots and dicots (Voegele et al. 2011). In addition to the phylogenetic separation of GID1ac and GID1b subfamilies, a number of important features related to the functional specialization of GID1 subfamilies have been described: 1) a remarkable difference in their transcriptional profiles across several tissues, such as in roots (Griffiths et al. 2006) and during germination (Bellieny-Rabelo et al. 2016); 2) the transcriptional down-regulation of GID1ac, but not GID1b, by GA. (Voegele et al. 2011); 3) The different affinity of GID1 subfamilies for GA, with GID1b displaying greater affinity for $\mathrm{GA}_{3}$ and GA4 than GID1a and GID1c (Nakajima et al. 2006) and; 4) The preference of specific GID1 proteins for particular DELLA groups (Hirano et al. 2007), potentially increasing the complexity involved in GA signaling.

Although important aspects of the GID1 family have been elucidated since its discovery and structural determination, important questions remain to be answered regarding GID1 expansion and diversification, the distribution of GID1ac and GID1b subfamilies in major eudicot lineages and the major evolutionary forces shaping the eudicot subfamilies at the sequence and expression levels. Here we performed a comprehensive survey of GID1 proteins in 52 plant genomes and integrate this data with protein structure and gene expression data. Our results provide important insights on the evolutionary history of the GID1 family in land plants, including findings such as: 1) a detailed phylogenetic reconstruction of GID1s and the identification of the main expansion and diversification events, including a potential contribution of whole-genome duplication (WGD) events to the structure of the GID1 family in eudicots; 2) the conservation and divergence of key amino acid residues involved in GA and DELLA binding 
by GID1b and GID1ac subfamilies and; 3) the important contribution of gene expression divergence in the establishment and divergence of the GID1ac and GID1b subfamilies in eudicots. Finally, we discuss theoretical aspects regarding the evolution of GA perception mechanisms, which that can fuel future computational and experimental studies.

\section{RESULTS AND DISCUSSION}

Expansion and diversification of GID1 receptors in major groups of land plants

A total of 52 diverse plant genomes, including angiosperms, gymnosperms and basal land plants (i.e. a lycophyte and a bryophyte) (Supplementary Table S1), were screened for GID1 proteins (see methods for details). Due to their high sequence similarity to HSLs, GID1s were separated with the aid of a phylogenetic reconstruction strategy (see methods for details) (Supplementary Fig. S1). We identified a total of 138 GID1 genes, with a median of two GID1s per genome (Supplementary Table S2) and $\sim 81 \%$ of the angiosperms containing 2-3 GID1 genes (Fig. 1). All eudicots except the early-branching Aquilegia coerulea have more than one GID1, which were classified using BLAST searches against Ar. thaliana GID1s and phylogenetic reconstructions by Bayesian and Maximum Likelihood approaches (see methods for details) (Fig. 2; Supplementary Table S2; Supplementary Fig. S2). The species with the greatest number of GID1s are Gossypium hirsutum (nine), Go. raimondii (six) and Glycine soja (six) in eudicots, and Musa acuminata (six) in monocots (Fig. 1). The 138 GID1s can be divided in four statistically supported groups (Voegele et al. 2011): group I (GID1ac) and II (GID1b), both with eudicot sequences; group III, with monocot GID1s and; group IV, containing GID1s from gymnosperms and basal plants. While GID1s from basal land plants and gymnosperms formed a separate small group, angiosperm GID1s diversified in the three former groups (Fig. 2). Our 
123

124

125

126

127

128

129

130

131

132

133

134

135

136

137

138

139

140

141

142

143

144

145

results support the monophyly of all plant GID1s, most likely from a single ancestral gene. The only GID1 from Amborella trichopoda (a basal angiosperm) is a sister group of the monocot and eudicot clades (Fig. 2), supporting the expansion and divergence of GID1s after the emergence of angiosperms. More precisely, this diversification process happened after the split of Ranuncales, since Aq. coerulea has only one GID1 that is an early branch in the GID1ac clade (Fig. 2). Our results also indicate that the GID1ac group is more closely related to the ancestral GID1, whereas the GID1b subfamily originated in eudicots after the separation of monocots, possibly via the gamma polyploidy, a whole genome triplication event shared by all core eudicots (Jiao et al. 2012). Conversely, monocot GID1s form a single clade without apparent strong diversification other than some recent lineage-specific expansions, particularly in banana (discussed below).

Next, we sought to explore the evolutionary history of the eudicot GID1ac and GID1b subfamilies. It is clear from our results that there is at least one GID1ac and one GID1b in every core eudicot, implying that these subfamilies acquired important non-redundant roles early in the evolution of eudicots (Voegele et al. 2011). Although GID1ac and GID1b subfamilies have comparable total sizes, their distribution is not uniform across lineages (Fig. 1). Asterids and many rosids have a single GID1ac, although some independent lineage-specific expansions happened after the separation of these two large groups (Fig. 1, Fig. 2). In Malvaceae, the two Gossypium species experienced a more recent GID1ac expansion, after the split from Theobroma cacao. In the order Brassicales, the GID1ac subfamily expanded after the separation of Brassicaceae and Caricaceae, with the emergence of a well-defined clade (harboring proteins related to Ar. thaliana GID1a) in the former, whereas Carica papaya preserved a single GID1c, outside of the GID1a clade (Fig. 2). In fact, all GID1a proteins belong to a Brassicaceae-specific 
monophyletic clade nested inside GID1c; this GID1a clade could have emerged at the WGD events that took place after the split of Brassicaceae and Caricaceae (Schranz 2006). Interestingly, with the exception of Capsella grandiflora and Capsella rubella (Fig. 2), the Brassicaceae species retained both GID1a and GID1c genes, indicating that they also play nonredundant roles (Suzuki et al. 2009). Nevertheless, it has been shown that GID1a and GID1c can compensate the absence of each other during Ar. thaliana seed germination (Voegele et al. 2011), suggesting that such non-overlapping roles are performed in other conditions/tissues (Griffiths et al. 2006). Capsella species are the only core eudicots without a classical GID1c, indicating a displacement of GID1c by GID1a in this genus. Therefore, these species would be good models to study the recent functional diversification within the GID1ac clade. Other GID1ac duplications that could be attributed to WGD events were also found in Salicaceae (Populus trichocarpa), Glycine, Manihot esculenta and in the most recent ancestor of Malus domestica and Pyrus x bretschneideri (Fig. 2). Further, all the Fabaceae species except Gl. max and Gl. soja have a single GID1ac. Our phylogenetic analysis indicates that one of the GID1ac paralogs was rapidly lost after the legume WGD and the remaining GIDlac gene was later duplicated at the Glycine WGD. This scenario is also supported by the presence of gene pairs with low Ks values the domesticated and wild soybeans (Fig. 2, Supplementary Table S3).

On the other hand, GID1b is mainly expanded in legumes, most likely due to the WGD events that happened at the base of Papilionoideae and Glycine. Except for Lotus japonicus and Cajanus cajan (which independently lost one GID1b paralog), all other legumes retained duplicated GID1b sets, with two duplication rounds accounting for the 3-4 GID1b genes found in soybeans (Fig. 1, Fig. 2). Similarly to what was observed for GID1ac, there is also a soybean GID1b pair (Gmax.GID1b1 and Gmax.GID1b2) that probably originated in the Glycine WGD 
(Fig. 2). This gene pair has a low Ks value (i.e. 0.187) that is compatible with the Glycine WGD age. Although these genes are not located in previously identified large homeologous segments (Severin et al. 2011), they show some level of conservation in their genomic neighborhood (Supplementary Fig. S3). Further, this scenario implies a loss of one GID1b in Gl. max after the separation from Gl. soja, possibly during soybean domestication; this hypothesis is supported by phylogenetic reconstructions (Fig. 1) and the low Ks values of the respective surviving Gl. soja paralogous pair (Gsoja.GIDIb3 and Gsoja.GIDIb4; Ks = 0.127; Fig. 2, Supplementary Table S3). Other expansions of GID1b genes can also be found in Manihot esculenta, Fragaria vesca, Populus, Solanum and Gossypium, for which polyploidization events have been documented or predicted (Mühlhausen and Kollmar 2013; Sato et al. 2012; Tuskan et al. 2006; Xu et al. 2011; Zhang et al. 2015). Conversely to what was observed in GID1ac, the GID1b subfamily size is constrained in Brassicales, in which only Brassica rapa has more than one gene, which may have originated by a recent Brassica whole-genome triplication event (Fig. 1, Fig. 2). Remarkably, even after two WGDs, Brassicaceae species have a single GID1b, indicating that the retention of GID1b duplicates is peculiar to a few clades, particularly legumes.

In monocots we have not found large and diversified GID1 subgroups (Fig. 1, Fig. 2), although there are multiple recent duplications in various lineages (e.g. maize and wheat). The most striking expansion of GID1 in monocots occurred in banana, in which six GID1s were found (Fig. 1, Fig. 2). Interestingly, although three recent WGDs have been identified in the banana genome (D'Hont et al. 2012), the Ks values of these GID1 pairs are far greater than expected for duplicates generated in these WGDs (Supplementary Table S3). The only banana GID1 pair with low Ks, Macum.GID1_2 and Macum.GID1_3, is separated by less than 20 kb, with a single intervening gene, supporting an origin via proximal (i.e. tandem) duplication. 
192 Furthermore, these banana GID1 pairs are outside of the homeologous blocks identified in the

193 banana genome project (D'Hont et al. 2012), suggesting that they originated by duplication

194 mechanisms other than the recent WGD.

195

GID1 intron-exon structure is largely conserved throughout the evolution of land plants

In addition to genomic locations and phylogenetic reconstructions, we also investigated 198 the GID1 gene architectures (i.e. intron-exon structures) and intron phases (Fig. 3, 199 Supplementary Fig. S4). There are three possible intron phases: phase 0, in which an intron is 200 located between two codons; phase 1 and 2, with introns between the first and second, and 201 between the second and third codon nucleotides, respectively. This analysis revealed a strong 202 conservation at the level of intron-exon structure. We found that 106 out of 126 angiosperm 203 GID1s $(\sim 84 \%)$ with available gene structure have the same basic gene structure, comprising a 204 short and a long exon (average length of 42 bp and $990 \mathrm{bp}$, respectively), with an intervening 205 phase 0 intron of $\sim 610$ bp (Fig. 3; Supplementary Fig. S4). Gene structure conservation is even greater in eudicots, which have 95 out of 108 genes $(88 \%)$ with the canonical architecture (Fig. 3). Remarkably, gene structure conservation in eudicots is independent of subfamily division, strongly supporting the evolution of eudicot GID1 subgroups from a single ancestor, most likely with the gene structure similar to that of Acoer.GID1. The canonical GID1 gene structure is also largely preserved in monocots, although three different architectures are found in banana GID1s

211 (Fig. 3). Importantly, the lycophyte GID1s resemble this architecture, indicating that it represents 212 an ancestral state that has been widely conserved throughout angiosperms. However, 213 gymnosperm and bryophyte GID1 gene architectures are distinct from this theme, as are those of 214 other few angiosperm GID1s. 


\section{GID1 subfamilies have shared and specific structural features}

Two critical steps in the evolution of GID1s from the HSL family were the loss of catalytic activity and the emergence of GA-binding properties (Hirano et al. 2012). We performed extensive sequence comparisons to better understand the conservation and divergence of GID1 subfamilies. Notably, the characteristic motifs HGGS and GDSSG are conserved in all analyzed GID1s, except for the presence of HGGG instead of HGGS and GDSAG instead of GDSSG in bryophytes) (Fig. 4). Moreover, a SUMO-Interaction Motif (SIM; amino acids $\mathrm{W}[\mathrm{V} / \mathrm{I}] \mathrm{LI}$ ), that is important for the recognition of SUMOylated DELLA proteins (Conti et al. 2014; Nelis et al. 2015), is also conserved across GID1s, again except in bryophytes. Five GID1s have amino acid substitutions in the first position of the SIM: Met (in Bdist.GID1 and Bstac.GID1), Tyr (in Mtrun.GID1b) and Phe (in Pgalu.GID1 and Ptaed.GID1). A hydrophobic surface in the N-terminal lid ( $\mathrm{Leu}^{18}, \mathrm{Trp}^{21}, \mathrm{Val}^{29}, \mathrm{Ile}^{33}, \mathrm{Leu}^{45}$ and $\mathrm{Tyr}^{48}$ in Ar. thaliana GID1a) forms a DELLA-binding surface (Murase et al. 2008; Shimada et al. 2008) and is also highly conserved in almost all GID1s (Fig. 4, Supplementary Fig. S5); we mapped these hydrophobic residues in the alignment and found that $\mathrm{Leu}^{45}$ is fully conserved, whereas the remaining positions tolerate substitutions by other hydrophobic residues (Fig. 4, Supplementary Fig. S5). For example, instead of $\mathrm{Ile}^{33}, \mathrm{Met}^{33}$ is present in all monocots (except rice) and in very few eudicots, mainly Solanaceae (i.e. Slyco.GID1b1, Slyco.GID1.b2, Stube.GID1.b1, Stube.GID1b2 and Achin.GID1b). Met ${ }^{33}$ seems to be the ancestral state, as it is present in Am. trichopoda and Aq. coerulea. Further, because these species have only a single GID1, we hypothesize that $\mathrm{Met}^{33}$ can be part of GID1 DELLA binding surfaces. Unexpectedly, some GA interacting residues (i.e. $\mathrm{Asn}^{218}, \mathrm{Phe}^{238}, \mathrm{Val}^{239}, \mathrm{Asp}^{243}, \mathrm{Arg}^{244}, \mathrm{Tyr}^{247}$ in $\mathrm{Ar}$. thaliana GID1a) are missing in all banana 
GID1s (Supplementary Fig. S5C). The impact of these mutations in the banana GID1s warrants further investigation, for example by expressing banana GID1s in rice GID1 mutants.

Although GID1s display an overall high level of sequence similarity, we were able to clearly define four major clades (Fig. 1), which support some level of functional divergence between them. To better understand the conservation patterns in the family, we sought to analyze conserved and specific motifs in GID1 subfamilies (Supplementary Fig. S5). We found five motifs that are conserved in all four clades (except in bryophyte GID1s, group IV). Three of those were well known motifs: Motif 1, which encompasses the SIM, GA- and DELLAinteracting residues; Motif 3, which contain the HGGS motif and; Motif 4 harbors the GDSSG domain and GA interacting residues. The remaining two motifs are Motif 5 and 6, which harbor other GA-binding residues (Supplementary Fig. S5). We also identified motifs specific to the GID1ac (Motif 2), GID1b (Motif 7) and monocot GID1s (Motif 8) sub-groups (Fig. 4, Supplementary Fig. S5). Further, these three motifs correspond to the same alignment region and their within-group conservation patterns suggest that they might play important subfamilyspecific roles.

To further explore the mechanistic differences of GID1ac and GID1b, we have also predicted functionally divergent sites using three different programs (see methods for details). A total of nine alignment positions were predicted to be functionally divergent between GID1ac and GID1b groups (Table 1). We mapped these residues on the tertiary structure of the Athal.GID1a (Fig. 5), as well as on the predicted conserved motifs described above. Two sites, Asp $^{102}$ and Gly ${ }^{103}$ in Athal.GID1a (Ser ${ }^{102}$ and $\mathrm{Thr}^{103}$ in Athal.GID1b) were inside the specific motifs discussed above (Fig. 4, Supplementary Fig. S5). Interestingly, the positions 102 and 103 are much more conserved in the GID1b (Ser ${ }^{102}$ and $\mathrm{Thr}^{103}$ in Athal.GID1b) than in the GID1ac 
260

261

262

263

264

265

266

267

268

269

270

271

272

273

274

275

276

277

278

279

280

281

282

283

subfamily (Fig. 4, Supplementary Fig. S5), supporting that these sites are under type I functional divergence ( $\mathrm{Gu} 2001,1999)$ (Table 1). Four other functionally divergent sites were highly conserved within GID1ac and GID1b subgroups but with important amino acid changes (e.g. Leu $^{323}$ in GID1ac subfamily and Phe ${ }^{323}$ in GID1b subfamily) between them, suggesting type II functional divergence (Table 1) (Gu 2001, 1999).

Intriguingly, one of the functionally divergent sites, Leu ${ }^{323}$ (in GID1ac; Phe ${ }^{323}$ in GID1b and $\mathrm{Leu}^{330}$ in rice GID1), is involved in hydrophobic interactions with GA (Murase et al. 2008).

Previous studies in rice demonstrated that mutation of GA interacting residues, including the substitution of $\mathrm{Leu}^{330}$ for $\mathrm{Il}^{330}$ or $\mathrm{Ala}^{330}$, reduced the GID1 affinity and specificity for GAs (Hirano et al. 2007; Shimada et al. 2008; Xiang et al. 2011). We performed in silico mutagenesis with FoldX to estimate the effects of converting $\mathrm{Leu}^{323}$ into $\mathrm{Phe}^{323}$ on the GA binding pocket of Athal.GID1a (2ZSH and 2ZSI), followed by docking analysis of mutated 2ZSH with $\mathrm{GA}_{3}$ and mutated 2ZSI with $\mathrm{GA}_{4}$. The native and mutant docked GID1-GAs had similar hydrogen bond lengths (Murase et al. 2008). In previously reported structures, the $07-2$ atom of $\mathrm{GA}_{3} / \mathrm{GA}_{4}$ formed a hydrogen bond to the $\mathrm{O} \gamma$ atom of $\mathrm{Ser}^{191}$ with a distance of $2.9 \AA$ (for $\mathrm{GA}_{3}$ ) and $3.2 \AA$ (for $\mathrm{GA}_{4}$ ) (Fig. 6A, Fig. 6C). These corresponding distances became longer $\left(3.5 \AA\right.$ for both $\mathrm{GA}_{3}$ and $\mathrm{GA}_{4}$ ), although within the range of hydrogen bonds (Fig. 6B, Fig. 6D). Interestingly, we found that $\mathrm{Phe}^{323}$ is closer to $\mathrm{GA}_{3} / \mathrm{GA}_{4}$ than $\mathrm{Leu}^{323}$ with a significant difference of $\sim 1 \AA$, suggesting that $P \mathrm{P}^{323}$ in GID1b confers a tighter binding pocket that could be related with the higher affinity of GID1b for $\mathrm{GA}_{3} / \mathrm{GA}_{4}$. Interestingly, this higher affinity of GID1b has been attributed to a partially closed configuration of the N-terminal lid. We hypothesize that Phe ${ }^{323}$ may also contribute to this phenomenon.

\section{GID1 subfamilies have substantial divergence in their expression patterns}


expression peak in in flowers (not observed in common bean) (Supplementary Fig. S7). 
(Bellieny-Rabelo et al. 2016), but not in Ar. thaliana (Voegele et al. 2011). Taken together, these

308

309

310

311

312

313

314

315

316

317

318

319

320

321

322

323

324

325

326

327

328

329

results indicate that the expression divergence of GID1b and GID1ac in seed development and germination occurred before the split of soybean and Medicago [ 52 MYA (Kumar et al. 2017)], but after the origin of Rosids. Nevertheless, this scenario will be clearer only when more gene expression data of GID1 genes during seed development and germination become available for other species.

Similarly to legumes, Athal.GIDIb is more expressed than Athal.GIDla and Athal.GIDIc in roots, whereas GID1ac expression is dominant in leaves, flowers and developing seeds (Supplementary Fig. S10). Thus, our results indicate that the specialization of GID1b towards roots, nodules and dry seeds support the scenario where GID1b, probably because of its higher affinity for GA, is particularly suitable for conditions of low GA concentrations and/or tissues with high GA sensitivity (Tanimoto 1987, 1994). It has been shown that GA regulates root elongation and thickening (Tanimoto and Hirano 2013). In root elongation, GA action specifically takes place at the endodermis (Ubeda-Tomás et al. 2008). In addition, GA also influences the number and length of root meristems (Tanimoto and Hirano 2013; Ubeda-Tomás et al. 2009). Thus, GID1b probably specialized to mediate GA signaling in eudicot roots in the presence of low hormone concentrations.

We have also investigated GID1 expression in monocots and in the lycophyte $\mathrm{Se}$. moellendorffii, in which there are often fewer GID1 genes and no family subdivision (Supplementary Fig. S11, S12 and S13). Interestingly, we found that GID1 is highly expressed in all tissues, with at least one GID1 gene expressed in high levels in roots. Collectively, our results show that the high expression of GID1 in roots dates back to the origin of the canonical GA perception system in lycophytes (Hirano et al. 2007; Nelson and Steber 2016), far earlier than the 
emergence of seed plants. In species without GID1 subfamilies (e.g. monocots and lycophytes), often harboring a single GID1 gene, this gene is expressed in almost all tissues and displays high expression in roots. We hypothesize that after the divergence of GID1ac and GID1b subfamilies, the former retained roles more related to the ancestral GA perception system (already present in lycophytes), and was later recruited to more modern features like seed germination. On the other hand, GID1b specialized in conditions of low GA concentrations (e.g. roots and germinating legume seeds) through biased gene expression and accumulation of mutations that increased its affinity for GA (Nakajima et al. 2006). Further, with GID1ac mediating canonical GA signaling, GID1b was also free to integrate alternative GA perception mechanisms, such as GAindependent DELLA binding and non-proteolytic GA signaling (Fuentes et al. 2012; Yamamoto et al. 2010).

Important aspects regarding the origin of GA perception system remain to be elucidated. While the lycophyte Se. moellendorffii and the bryophyte Physcomitrella patens have the key components of the canonical GA perception machinery, such system seems to be absent in the latter (Hirano et al. 2007; Nelson and Steber 2016), which is supported by several lines of evidence (Hayashi et al. 2010; Vesty et al. 2016; Yasumura et al. 2007), such as: 1) Physcomitrella patens GID1 and DELLA do not interact; 2) Physcomitrella patens GID1 does not interact with GA; 3) DELLA-deficient $P$. patens strains do not exhibit derepressed growth like that observed in DELLA-deficient angiosperms; 4) Physcomitrella patens DELLA does not suppress GA response in rice, although it can repress growth in Ar. thaliana. On the other hand, certain bioactive diterpene hormones from early steps of the GA biosynthesis pathway (e.g. entkaurene) promote spore germination in Physcomitrella patens (Hayashi et al. 2010; Vesty et al. 2016). Interestingly, ABA can inhibit Physcomitrella patens spore germination, strongly 
supporting the existence of a diterpene/ABA signaling module before the emergence of vascular plants, although apparently not as prominent as that found in seed plants (Hayashi et al. 2010). The key genes involved in diterpene perception in Physcomitrella patens remain to be elucidated and could involve direct diterpene recognition by GRAS domain proteins (e.g. DELLA), which were already diversified early in the evolution of land plants (Zhang et al. 2012).

\section{MATERIALS AND METHODS}

\section{Identification of GID1 proteins in angiosperms}

To identify the GID1 proteins in land plants, predicted proteins of 47 angiosperms, two gymnosperms, one lycophyte and one bryophyte were downloaded from various sources (Supplementary Table 1). GID1 homologs were identified in four steps: 1) BLASTP (Altschul et al. 1997) searches using experimentally characterized GID1s from Ar. thaliana, Lepidium sativum and rice to search the predicted proteomes of each species (a total 1,995,759 proteins), with e-value and similarity thresholds of $\leq 1 \mathrm{e}^{-5}$ and $\geq 38 \%$, respectively. This step resulted in a total of 252 proteins; 2) Only the 238 sequences with the conserved motifs HGG and GXSXG, also shared with HSLs and other plant carboxylesterases (Ueguchi-Tanaka et al. 2005; Voegele et al. 2011), were retained; 3) Bona-fide GID1s were separated from plant carboxylesterases using a phylogenomic approach, as follows: carboxylesterases of Ar. thaliana (AT5G23530) and rice (ABA92266) (Hirano et al. 2007) were aligned with the 238 GID1 candidates using PROMALS3D (Pei et al. 2008). The phylogenetic reconstruction was performed with FastTree (Price et al. 2010). A total of 138 GID1s clearly clustered in a monophyletic clade (Supplementary Fig. S1) and were separated from carboxylesterases; 4) redundancy was removed with the aid of BLASTCLUST (95\% coverage and 95\% identity thresholds) (Altschul et al. 1997). These steps allowed us to identify 130 GID1s. We found only one GID1 in Vitis 
377

378

379

380

381

382

383

384

385

386

387

388

389

390

vinifera, but two V. vinifera GID1 genes AM479851 and AM468374 were available in Genbank and were used. Our collection was supplemented with Triticum aestivum and L. sativum GID1s (three from each) (Li et al. 2013; Voegele et al. 2011). One GID1 from Ca. cajan was excluded because of the absence of a start codon. The coding sequences of the identified GID1s were also searched in their respective genomes using BLASTN with an e-value threshold of $\leq 1 \mathrm{e}^{-6}$ (Altschul et al. 1997), which allowed us to identify an additional Gl. soja GID1.

We have not found GID1 genes in the Picea glauca, probably due to genome incompleteness. One of identified GID1 genes of Se. moellendorffi was fragmented. We obtained GID1 sequences from Pi. glauca and Se. moellendorffi from individual Genbank entries [Pi. glauca (Genbank: BN001188.1) and Se. moellendorffii (Refseq: XP_002993392.1, XP_002993392.1)]. Overall, a total of 138 GID1s were used in the analyses (Supplementary Table S2). Species names were abbreviated by the first letter of genus followed by the four letters of the species name (e.g. Athal corresponds to Ar. thaliana) (Supplementary Table S2). Eudicot GID1s were classified in GID1a, GID1b and GID1c using Ar. thaliana GID1s as reference. Non-eudicot GID1s were simply numbered, as there is no subfamily division in these species.

\section{Sequence analysis and phylogenetic reconstruction}

Multiple sequence alignment of GID1 proteins was carried out using PROMALS3D (Pei et al. 2008) and visually inspected with Jalview (Waterhouse et al. 2009). Large N- and Cterminal gaps were removed. Conserved motifs were analyzed with MEME (Bailey et al. 2009). Phylogenetic reconstructions were performed with MrBayes (v3.2.2; 3,000,000 generations, sampling frequency: 100) (Ronquist and Huelsenbeck 2003). Convergence was assessed using 
400

401

402

403

404

405

406

407

408

409

410

411

412

413

414

415

416

417

418

419

420

421

422

423

Tracer (v1.6) (http://beast.bio.ed.ac.uk/Tracer) and posterior probabilities were estimated by removing the burn-in generations as required. Alternatively, we have also reconstructed phylogenies using RAxML (best fit model, 100 bootstrap samples) (Stamatakis 2014). Gene structure analysis was performed using GSDS (v2) (Hu et al. 2015). The aligned proteins were used to guide the conversion of cDNA into codon alignments by PAL2NAL (Suyama et al. 2006). CODEML (PAML version 4.9b) (Yang 1997) was used in for Ks calculation. The Goldman and Yang ML method and the F3x4 model were applied.

\section{Functional divergence, in silico mutagenesis and docking}

We used three different programs to infer functionally divergent sites of GID1ac and GID1b subfamilies (with default options): FunDi (Gaston et al. 2011), GroupSim (Capra and Singh 2008) and Sequence Harmony (Feenstra et al. 2007). We used a threshold of 0.5 (Gaston et al. 2011) to filter the sites identified by FunDi and GroupSim, and the default threshold in Sequence harmony. Only those sites identified by all three programs were considered. An in silico mutagenesis approach was performed with FoldX (Schymkowitz et al. 2005), using the wild type crystal structures 2ZSH (GA 3 -GID1a-DELLA) and 2ZSI (GA4-GID1a-DELLA), excluding $\mathrm{GA}_{3}$, GA 4 and DELLA. Crystal structures 2ZSH and 2ZSI were downloaded from the PDB database (Berman et al. 2000). Protein-ligand docking was performed using SwissDock (Grosdidier et al. 2011) and ligands ( $\mathrm{GA}_{3}$ and $\left.\mathrm{GA}_{4}\right)$ were selected from the ZINC database (Irwin and Shoichet 2006). All structures were visualized by PyMOL (http://www.pymol.org/).

\section{Gene expression data}

Gene expression data of GID1 genes were obtained from publicly available sources, as following. Soybean: Soybase (http://soybase.org/soyseq/) (Severin et al. 2010) and from a recent 
manuscript from our group (Bellieny-Rabelo et al. 2016). Phaseolus vulgaris: Common bean gene expression atlas (http://plantgrn.noble.org/PvGEA/) (O'Rourke et al. 2014). Me. truncatula: Medicago truncatula Gene Expression Atlas (MtGEA) (Benedito et al. 2008). Ar. thaliana: AtGenExpress (Schmid et al. 2005). Rice: Rice Express ion Database; (http://expression.ic4r.org). Maize and Se. moellendorffii gene expression data were obtained from recent publications (Stelpflug et al. 2016; Zhu et al. 2017).

\section{REFERENCES}

Aleman L, et al. 2008. Functional analysis of cotton orthologs of GA signal transduction factors GID1 and SLR1. Plant Molecular Biology 68: 1-16. doi: 10.1007/s11103-008-9347-z

Altschul SF, et al. 1997. Gapped BLAST and PSI-BLAST: a new generation of protein database search programs. Nucleic Acids Research 25: 3389-3402.

Bailey TL, et al. 2009. MEME Suite: Tools for motif discovery and searching. Nucleic Acids Research 37: 202-208. doi: 10.1093/nar/gkp335

Bellieny-Rabelo D, et al. 2016. Transcriptome analysis uncovers key regulatory and metabolic aspects of soybean embryonic axes during germination. Scientific Reports 6: 36009. doi: 10.1038/srep36009

Benedito VA, et al. 2008. A gene expression atlas of the model legume Medicago truncatula. Plant Journal 55. doi: 10.1111/j.1365-313X.2008.03519.x

Berman HM, et al. 2000. The protein data bank. Nucleic Acids Research 28. doi: 10.1093/nar/28.1.235

Capra JA, Singh M 2008. Characterization and prediction of residues determining protein functional specificity. Bioinformatics 24. doi: 10.1093/bioinformatics/btn214

Chandler PM, et al. 2008. Characterization of gibberellin receptor mutants of barley (Hordeum vulgare L.). Molecular Plant 1: 285-294. doi: 10.1093/mp/ssn002

Conti L, et al. 2014. Small Ubiquitin-like Modifier Protein SUMO Enables Plants to Control Growth Independently of the Phytohormone Gibberellin. Developmental Cell 28: 102-110. doi: 10.1016/j.devcel.2013.12.004

D'Hont A, et al. 2012. The banana (Musa acuminata) genome and the evolution of monocotyledonous plants. Nature 488: 213-217. doi: 10.1038/nature11241

Dill A, Thomas SG, Hu J, Steber CM, Sun T-P 2004. The Arabidopsis F-box protein SLEEPY1 targets gibberellin signaling repressors for gibberellin-induced degradation. The Plant Cell 16: 1392-1405. doi: 10.1105/tpc.020958

Feenstra KA, Pirovano W, Krab K, Heringa J 2007. Sequence harmony: detecting functional specificity from alignments. Nucleic Acids Research 35. doi: 10.1093/nar/gkm406

Fleet CM, Sun TP 2005. A DELLAcate balance: The role of gibberellin in plant morphogenesis. Current Opinion in Plant Biology 8: 77-85. doi: 10.1016/j.pbi.2004.11.015

Fu X, et al. 2004. The Arabidopsis mutant sleepy1 gar2-1 protein promotes plant growth by increasing the affinity of the SCF SLY1 E3 ubiquitin ligase for DELLA protein substrates. The Plant Cell 16: 14061418. doi: 10.1105/tpc.021386.dependent 
Fuentes S, et al. 2012. Fruit Growth in Arabidopsis Occurs via DELLA-Dependent and DELLA-Independent Gibberellin Responses. The Plant Cell 24: 3982-3996. doi: 10.1105/tpc.112.103192

Gaston D, Susko E, Roger AJ 2011. A phylogenetic mixture model for the identification of functionally divergent protein residues. Bioinformatics 27: 2655-2663. doi: 10.1093/bioinformatics/btr470

Gomi K, et al. 2004. GID2, an F-box subunit of the SCF E3 complex, specifically interacts with phosphorylated SLR1 protein and regulates the gibberellin-dependent degradation of SLR1 in rice. Plant Journal 37: 626-634. doi: 10.1111/j.1365-313X.2003.01990.x

Griffiths J, et al. 2006. Genetic characterization and functional analysis of the GID1 gibberellin receptors in Arabidopsis. The Plant Cell 18: 3399-3414. doi: 10.1105/tpc.106.047415

Grosdidier Al, Zoete V, Michielin O 2011. SwissDock, a protein-small molecule docking web service based on EADock DSS. Nucleic Acids Research 39: 270-277. doi: 10.1093/nar/gkr366

Gu X 2001. Maximum-likelihood approach for gene family evolution under functional divergence. Molecular Biology and Evolution 18.

Gu X 1999. Statistical methods for testing functional divergence after gene duplication. Molecular Biology and Evolution 16.

Hauvermale AL, Tuttle KM, Takebayashi Y, Seo M, Steber CM 2015. Loss of Arabidopsis thaliana Seed Dormancy is Associated with Increased Accumulation of the GID1 GA Hormone Receptors. Plant and Cell Physiology. doi: 10.1093/pcp/pcv084

Hayashi K-i, et al. 2010. Endogenous Diterpenes Derived from ent-Kaurene, a Common Gibberellin Precursor, Regulate Protonema Differentiation of the Moss Physcomitrella patens. PLANT PHYSIOLOGY 153: 1085-1097. doi: 10.1104/pp.110.157909

Hirano K, Aya K, Matsuoka M, Ueguchi-Tanaka M 2012. Molecular Determinants that Convert Hormone Sensitive Lipase into Gibberellin Receptor. Protein and Peptide Letters 19: 180-185. doi: $10.2174 / 092986612799080248$

Hirano K, et al. 2007. The GID1-mediated gibberellin perception mechanism is conserved in the Lycophyte Selaginella moellendorffii but not in the Bryophyte Physcomitrella patens. The Plant Cell 19: 3058-3079. doi: 10.1105/tpc.107.051524

$\mathrm{Hu}$ B, et al. 2015. GSDS 2.0: An upgraded gene feature visualization server. Bioinformatics 31: 12961297. doi: 10.1093/bioinformatics/btu817

Irwin JJ, Shoichet BK 2006. ZINC - A Free Database of Commercially Available Compounds for Virtual Screening. J. Chem. Inf. Model 45: 177-182.

luchi S, et al. 2007. Multiple loss-of-function of Arabidopsis gibberellin receptor AtGID1s completely shuts down a gibberellin signal. Plant Journal 50: 958-966. doi: 10.1111/j.1365-313X.2007.03098.x

Jiao Y, et al. 2012. A genome triplication associated with early diversification of the core eudicots. Genome Biology 13: R3. doi: 10.1186/gb-2012-13-1-r3

Kumar S, Stecher G, Suleski M, Hedges SB 2017. TimeTree: A Resource for Timelines, Timetrees, and Divergence Times. Molecular Biology and Evolution 34: 1812-1819. doi: 10.1093/molbev/msx116

Li A, et al. 2013. Molecular characterization of three GIBBERELLIN-INSENSITIVE DWARF1 homologous genes in hexaploid wheat. Journal of Plant Physiology 170: 432-443. doi: 10.1016/j.jplph.2012.11.010

Livne S, Weiss D 2014. Cytosolic activity of the gibberellin receptor GIBBERELLIN INSENSITIVE DWARF1A. Plant and Cell Physiology 55: 1727-1733. doi: 10.1093/pcp/pcu104

McGinnis KM, et al. 2003. The Arabidopsis SLEEPY1 gene encodes a putative F-box subunit of an SCF E3 ubiquitin ligase. The Plant Cell 15: 1120-1130. doi: 10.1105/tpc.010827.increased

Mühlhausen S, Kollmar M 2013. Whole genome duplication events in plant evolution reconstructed and predicted using myosin motor proteins. BMC Evolutionary Biology 13. doi: 10.1186/1471-2148-13202 
Murase K, Hirano Y, Sun T-p, Hakoshima T 2008. Gibberellin-induced DELLA recognition by the gibberellin receptor GID1. Nature 456: 459-463. doi: 10.1038/nature07519

Nakajima M, et al. 2006. Identification and characterization of Arabidopsis gibberellin receptors. Plant Journal 46: 880-889. doi: 10.1111/j.1365-313X.2006.02748.x

Nelis S, Conti L, Zhang C, Sadanandom A 2015. A functional Small Ubiquitin-like Modifier (SUMO) interacting motif (SIM) in the gibberellin hormone receptor GID1 is conserved in cereal crops and disrupting this motif does not abolish hormone dependency of the DELLA-GID1 interaction. Plant signaling \& behavior 10: e987528. doi: 10.4161/15592324.2014.987528

Nelson SK, Steber CM. 2016. Gibberellin hormone signal perception: down-regulating DELLA repressors of plant growth and development. In. Annual Plant Reviews, Volume 49: John Wiley \& Sons, Ltd. p. 153-188.

O'Rourke Ja, et al. 2014. An RNA-Seq based gene expression atlas of the common bean. BMC Genomics 15. doi: 10.1186/1471-2164-15-866

Olszewski N, Sun T-P, Gubler F 2002. Gibberellin signaling: biosynthesis, catabolism, and response pathways. The Plant Cell 14 Suppl: S61-S80. doi: 10.1105/tpc.010476.GAs

Pei J, Kim B-H, Grishin NV 2008. PROMALS3D: a tool for multiple protein sequence and structure alignments. Nucleic Acids Research 36: 2295-2300. doi: 10.1093/nar/gkn072

Peng J, et al. 1997. The Arabidopsis GAI gene defines a signaling pathway that negatively regulates gibberellin responses. Genes and Development 11: 3194-3205. doi: 10.1101/gad.11.23.3194

Price MN, Dehal PS, Arkin AP 2010. FastTree 2 - Approximately maximum-likelihood trees for large alignments. PLoS ONE 5. doi: 10.1371/journal.pone.0009490

Ronquist F, Huelsenbeck JP 2003. MrBayes 3: Bayesian phylogenetic inference under mixed models. Bioinformatics 19: 1572-1574. doi: 10.1093/bioinformatics/btg180

Sato $S$, et al. 2012. The tomato genome sequence provides insights into fleshy fruit evolution. Nature 485: 635-641. doi: 10.1038/nature11119

Schmid M, et al. 2005. A gene expression map of Arabidopsis thaliana development. Nature Genetics 37: 501-506. . doi: 10.1038/ng1543

Schranz ME 2006. Independent Ancient Polyploidy Events in the Sister Families Brassicaceae and Cleomaceae. THE PLANT CELL ONLINE 18: 1152-1165. doi: 10.1105/tpc.106.041111

Schymkowitz J, et al. 2005. The FoldX web server: an online force field. Nucleic Acids Research 33: $382-$ 388. doi: 10.1093/nar/gki387

Severin AJ, Cannon SB, Graham MM, Grant D, Shoemaker RC 2011. Changes in twelve homoeologous genomic regions in soybean following three rounds of polyploidy. The Plant Cell 23: 3129-3136. doi: 10.1105/tpc.111.089573

Severin AJ, et al. 2010. RNA-Seq Atlas of Glycine max: a guide to the soybean transcriptome. BMC plant biology 10. doi: 10.1186/1471-2229-10-160

Shimada A, et al. 2008. Structural basis for gibberellin recognition by its receptor GID1. Nature 456: 520523. doi: $10.1038 /$ nature 07546

Stamatakis A 2014. RAxML version 8: a tool for phylogenetic analysis and post-analysis of large phylogenies. Bioinformatics 30: 1312-1313. doi: 10.1093/bioinformatics/btu033

Stelpflug SC, et al. 2016. An Expanded Maize Gene Expression Atlas based on RNA Sequencing and its Use to Explore Root Development. plant genome. doi: 10.3835/plantgenome2015.04.0025

Suyama M, Torrents D, Bork P 2006. PAL2NAL: Robust conversion of protein sequence alignments into the corresponding codon alignments. Nucleic Acids Research 34: 609-612. doi: 10.1093/nar/gkl315

Suzuki H, et al. 2009. Differential expression and affinities of Arabidopsis gibberellin receptors can explain variation in phenotypes of multiple knock-out mutants. Plant Journal 60: 48-55. doi: 10.1111/j.1365-313X.2009.03936.x 
Tanimoto E 1987. Gibberellin-Dependent Root Elongation in Lactuca sativa Recovery from Growth Retardant-Suppressed Elongation with Thickening by Low concentration of GA3. Plant and Cell Physiology 28: 963-973. doi: 10.1093/oxfordjournals.pcp.a077399

Tanimoto E 1994. Interaction of Gibberellin A3 and Ancymidol in the Growth and Cell-Wall Extensibility of Dwarf Pea Roots. Plant and Cell Physiology 35: 1019-1028. doi: 10.1093/oxfordjournals.pcp.a078689

Tanimoto E, Hirano K. 2013. Role of Gibberellins in Root Growth. In. Plant Roots: The Hidden Half, Fourth Edition. Boca Raton, FL, USA: CRC Press. p. 13-11--13-14.

Tuskan GA, et al. 2006. The Genome of Black Cottonwood, Populus trichocarpa (Torr. \&amp; Gray). Science 313: 1596-1604. doi: 10.1126/science.1128691

Ubeda-Tomás S, et al. 2009. Gibberellin Signaling in the Endodermis Controls Arabidopsis Root Meristem Size. Current Biology 19: 1194-1199. doi: 10.1016/j.cub.2009.06.023

Ubeda-Tomás S, et al. 2008. Root growth in Arabidopsis requires gibberellin/DELLA signalling in the endodermis. Nature Cell Biology 10: 625-628. doi: 10.1038/ncb1726

Ueguchi-Tanaka M, et al. 2005. GIBBERELLIN INSENSITIVE DWARF1 encodes a soluble receptor for gibberellin. Nature 437: 693-698. doi: 10.1038/nature04028

Vesty EF, et al. 2016. The decision to germinate is regulated by divergent molecular networks in spores and seeds. New Phytologist 211. doi: 10.1111/nph.14018

Voegele A, Linkies A, Muller K, Leubner-Metzger G 2011. Members of the gibberellin receptor gene family GID1 (GIBBERELLIN INSENSITIVE DWARF1) play distinct roles during Lepidium sativum and Arabidopsis thaliana seed germination. Journal of Experimental Botany 62: 5131-5147. doi: 10.1093/jxb/err214

Waterhouse AM, Procter JB, Martin DMA, Clamp M, Barton GJ 2009. Jalview Version 2-A multiple sequence alignment editor and analysis workbench. Bioinformatics 25: 1189-1191. doi: 10.1093/bioinformatics/btp033

Willige $B C$, et al. 2007. The DELLA domain of GA INSENSITIVE mediates the interaction with the GA INSENSITIVE DWARF1A gibberellin receptor of Arabidopsis. The Plant Cell 19: 1209-1220. doi: 10.1105/tpc.107.051441

Xiang $\mathrm{H}$, et al. 2011. Thermodynamic characterization of OsGID1-gibberellin binding using calorimetry and docking simulations. J Mol Recognit: 275-282. doi: 10.1002/jmr.1049

Xu X, et al. 2011. Genome sequence and analysis of the tuber crop potato. Nature 475: 189-195. doi: 10.1038/nature10158

Yamamoto $Y$, et al. 2010. A rice gid1 suppressor mutant reveals that gibberellin is not always required for interaction between its receptor, GID1, and DELLA proteins. The Plant Cell 22: 3589-3602. doi: 10.1105/tpc.110.074542

Yang Z 1997. PAML: a program package for phylogenetic analysis by maximum likelihood. Bioinformatics 13: 555-556. doi: 10.1093/bioinformatics/13.5.555

Yasumura Y, Crumpton-Taylor M, Fuentes S, Harberd NP 2007. Step-by-Step Acquisition of the Gibberellin-DELLA Growth-Regulatory Mechanism during Land-Plant Evolution. Current Biology 17. doi: 10.1016/j.cub.2007.06.037

Zhang D, lyer LM, Aravind L 2012. Bacterial GRAS domain proteins throw new light on gibberellic acid response mechanisms. Bioinformatics 28: 2407-2411. doi: 10.1093/bioinformatics/bts464

Zhang T, et al. 2015. Sequencing of allotetraploid cotton (Gossypium hirsutum L. acc. TM-1) provides a resource for fiber improvement. Nature Biotechnology 33: 531-537. doi: 10.1038/nbt.3207

Zhu Y, et al. 2017. Global transcriptome analysis reveals extensive gene remodeling, alternative splicing and differential transcription profiles in non-seed vascular plant Selaginella moellendorffii. BMC Genomics 18: 1-15. doi: 10.1186/s12864-016-3266-1 
Table 1. Number of sites predicted to be functionally divergent in GID1ac and GID1b groups.

606

\begin{tabular}{|c|c|c|c|}
\hline $\begin{array}{l}\text { Position in GID1a } \\
\text { crystal structure }\end{array}$ & $\begin{array}{c}\text { GID1ac group (Athal.GID1a } \\
\text { as reference) }\end{array}$ & $\begin{array}{c}\text { GID1b group (Athal.GID1b } \\
\text { as reference) }\end{array}$ & $\begin{array}{c}\text { Functional Divergence } \\
\text { Type }\end{array}$ \\
\hline Loop & $\mathrm{Asn}^{58}$ & $\mathrm{Phe}^{58}$ & Type I \\
\hline Loop & $\mathrm{Asp}^{102}$ & $\operatorname{Ser}^{102}$ & Type I \\
\hline Loop & Gly $^{103}$ & $\operatorname{Thr}^{103}$ & Type I \\
\hline Loop & $\mathrm{Ala}^{150}$ & $\operatorname{Ser}^{150}$ & Type II \\
\hline Loop & Lys $^{178}$ & Gly $^{178}$ & Type I \\
\hline a3 helix & Gly $^{205}$ & $\mathrm{Thr}^{205}$ & Type U \\
\hline Loop & $\mathrm{Asn}^{218}$ & $\mathrm{His}^{218}$ & Type II \\
\hline ๆ2 helix & $\mathrm{Leu}^{323}$ & $\mathrm{Phe}^{323}$ & Type II \\
\hline$\alpha 7$ helix & $\mathrm{Val}^{333}$ & $\mathrm{Leu}^{333}$ & Type II \\
\hline
\end{tabular}

607

608

609

610

611

612

613

614

615

616

617

618

619

620

621 


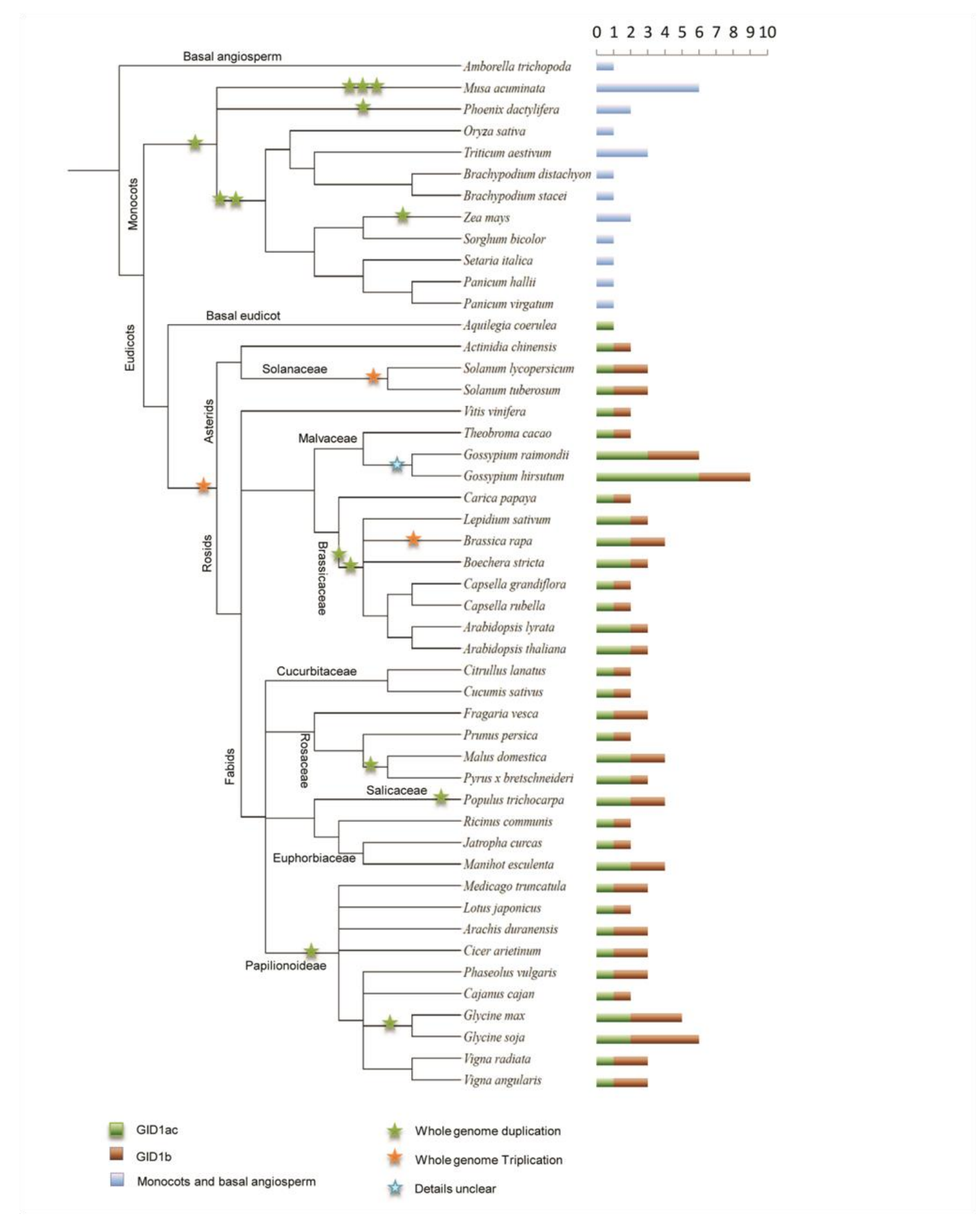

624 Fig. 1. Number of GID1 genes in different angiosperms. GID1 counts in each species are 625 represented as horizontal bars, colored according to subfamily. Polyploidization events are marked with colored stars. Species tree was generated using PhyloT (http://phylot.biobyte.de/). Branch lengths do not represent evolutionary time. 
bioRxiv preprint doi: https://doi.org/10.1101/221937; this version posted November 22, 2017. The copyright holder for this preprint (which was

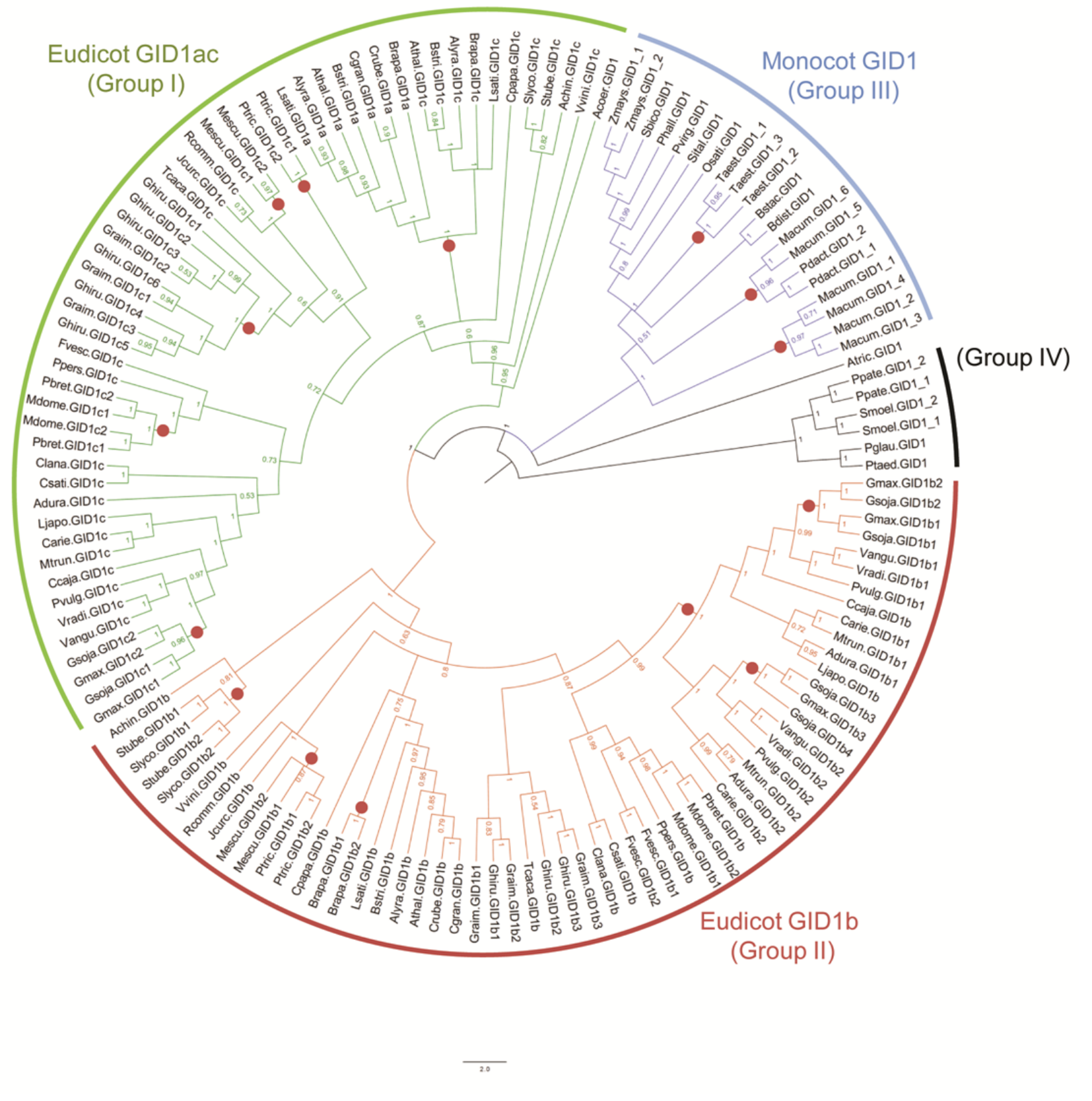

Fig. 2. Phylogenetic reconstruction of the 138 GID1 proteins identified in 52 plant species. Multiple sequence alignment of GID1 proteins was carried with PROMALS3D and phylogenetic reconstruction performed with MrBayes. The GID1 proteins were classified in four groups, which are represented in different colors. Red circles show genes potentially originated by whole-genome duplication events. 


\section{Group}

(I)

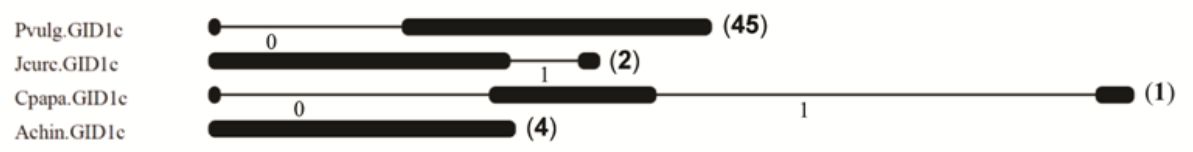

(II)

Mtrun.GIDIbl

Fvesc.GIDlbl

Pbret.GIDIb

Athal.GIDIb

Fvesc.GID1b2

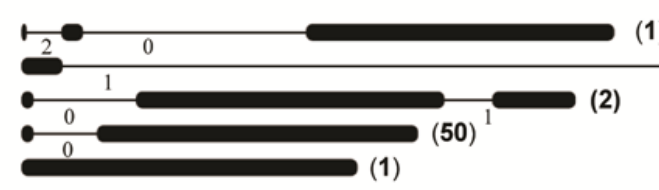

(1)

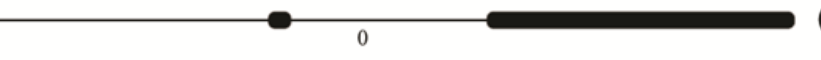

(III)

Osati.GID1

Pvirg.GIDI

Macum.GIDI_I

Macum.GIDI_3

Macum.GIDI_2

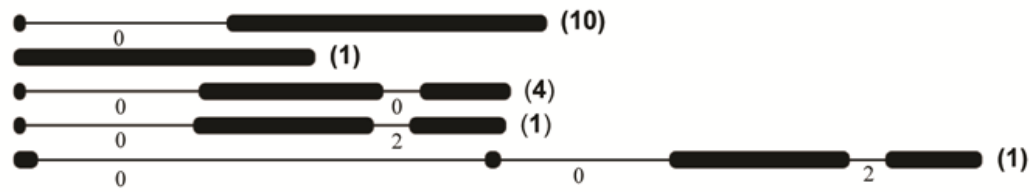

Basal angiosperm

Atric.GID1

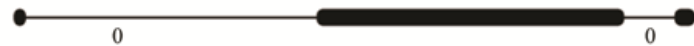

(IV)

Pglau.GID1
Ppate.GID1_1
Smoel.GID1_1
Smoel.GID1_2

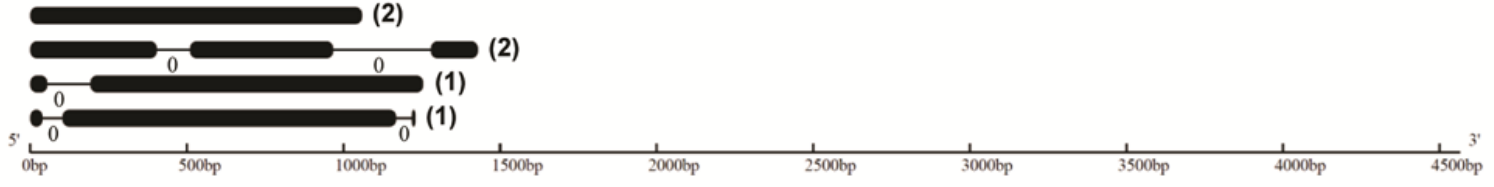

Fig. 3. Diagram of representative GID1 intron-exon architectures. Thin lines and thick bars represent introns and exons, respectively. Numbers below introns and at the right side of the gene architectures represent intron phases and number of occurrences of each structure, respectively. For comparison purposes, the intron-exon structure of the Am. trichopoda GID1, a 


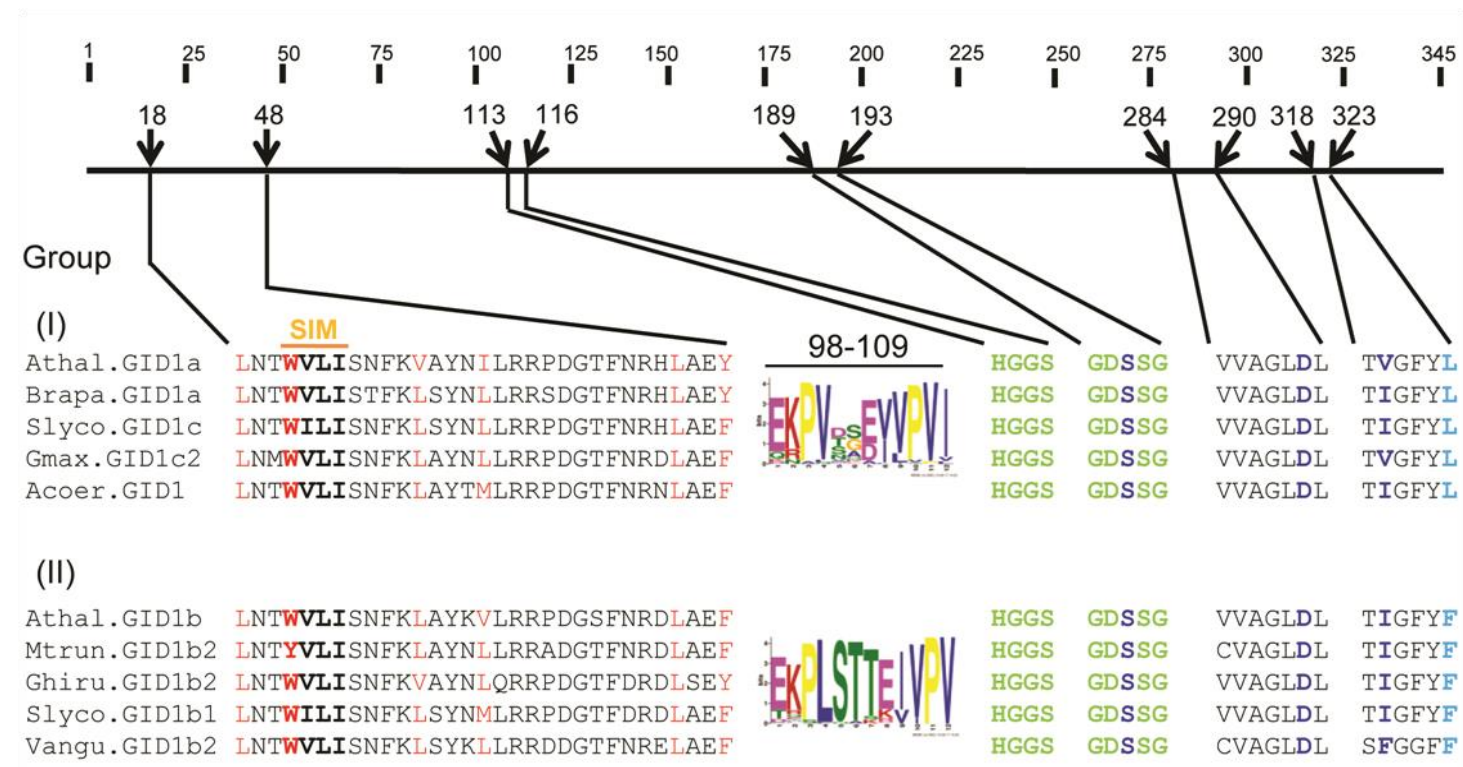

(III)

$\begin{array}{lllll}\text { Osati.GID1 } & \text { LHTWVLISNFKLSYNILRRADGTFERDLGEY } \\ \text { Bstac.GID1 } & \text { ITTMVISNFKVAYNMLRRPDGTFDRDLAEF } \\ \text { Phall.GID1 } & \text { IHTWVLISNFKLAYNMLRRADGTFDRDLAEY }\end{array}$

$\begin{array}{ll}\text { Ptaed.GID1 } & \text { LNTFVLISNFKLVYNLLRRPDGTFNRHLAEF } \\ \text { Smole.GID1_2 } & \text { LNTWVISISNLKLAYNLTRNSDGSFNRNLDEF } \\ \text { Ppate.GID1_1 } & \text { VQLRILSEFVVRANRVTRRRDGTINRWLADT } \\ \text { Ppate.GID1_2 } & \text { MQLRLLCKVVVKANDLARRKDGTINRWLADV } \\ & \end{array}$

DELLĀ binding

$\begin{array}{llll}\text { HGGS } & \text { GDSSG } & \text { VVAGLDL } & \text { TIGFYL } \\ \text { HGGS } & \text { GDSSG } & \text { VVAGLDM } & \text { TVGFFI } \\ \text { HGGG } & \text { GDASG } & \text { IVGGLDI } & \text { IHTFAL } \\ \text { HGGG } & \text { GDASG } & \text { IVGGLDI } & \text { IHTFAL }\end{array}$

$\alpha / \beta$-hydrolase fold domain $\overline{\mathrm{GA}} \overline{\text { binding pocket }}$

644 Fig. 4. Schematic representation of archetypal GID1s. The thick line with coordinates 645 represents the positions of important GID1 features, using the Ar. thaliana GID1a as a reference. 646 HGGS and GDSSG motifs are shown in green; the SIM motif (W[V/I]LI) is also tagged. 647 DELLA binding residues are shown in red and the 'catalytic triad' involved in binding GA (Ser, 648 Asp, and Val/Ile) are highlighted in dark blue. Subgroup specific motifs are represented with 649 logos. One functionally divergent site between GID1ac and GID1b, which is also a GA 650 interacting residue, is marked with sky blue color. All functionally divergent sites can be seen in 651 Supplementary Fig. S5 and Table 1. 
A)

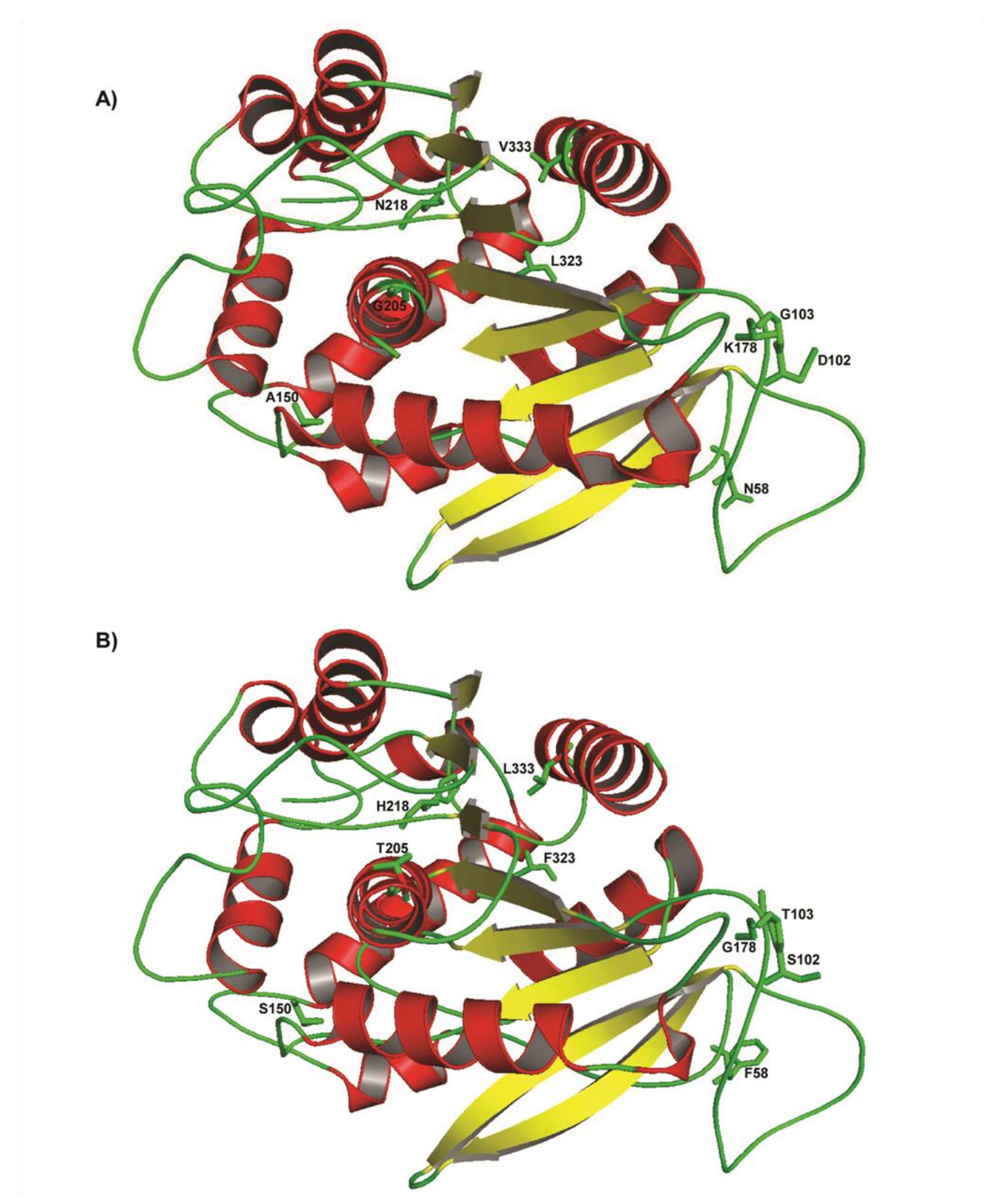

654 Fig. 5. Localization of critical amino acids in the 3D structures. Functionally divergent sites 655 were mapped on the crystal structure (as sticks) of the native (A) and mutated (in silico) (B) Ar. 656 thaliana GID1a (2ZSH). 
A)

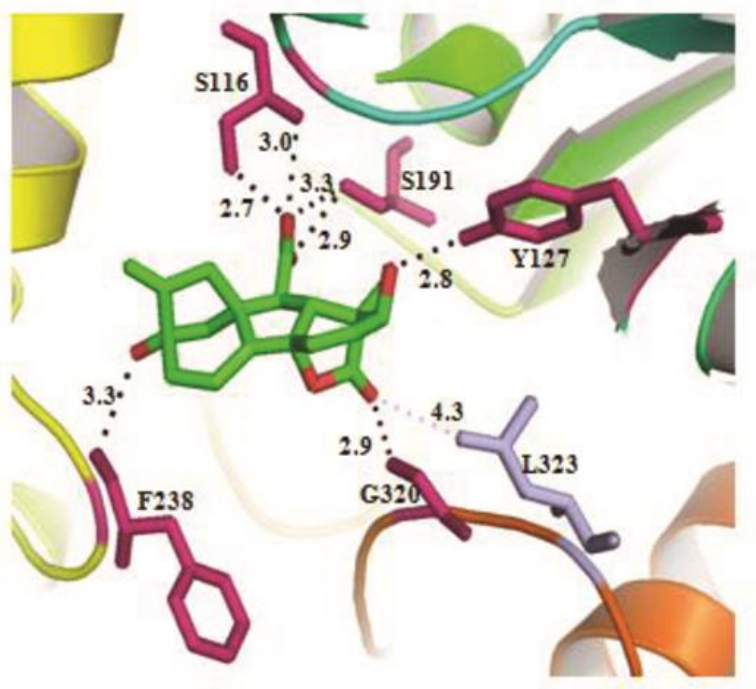

C)

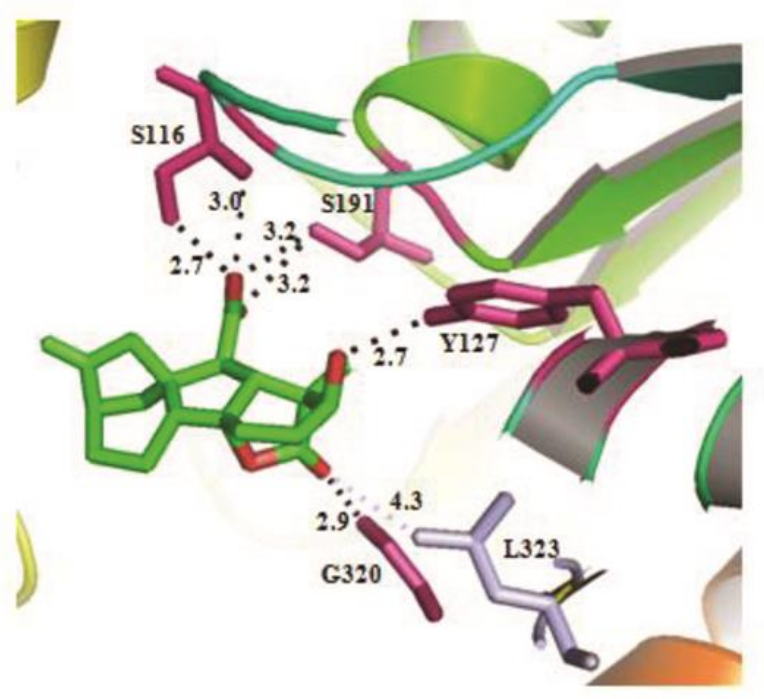

B)

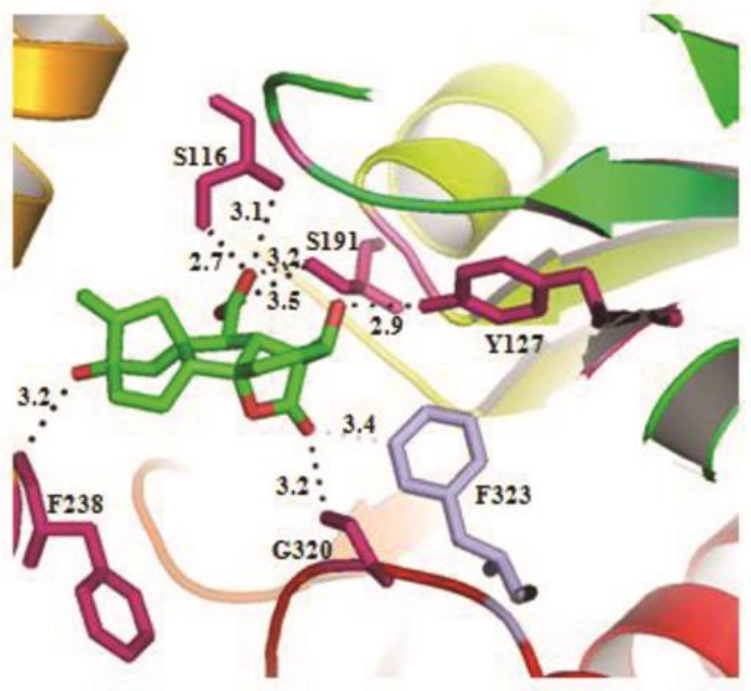

D)
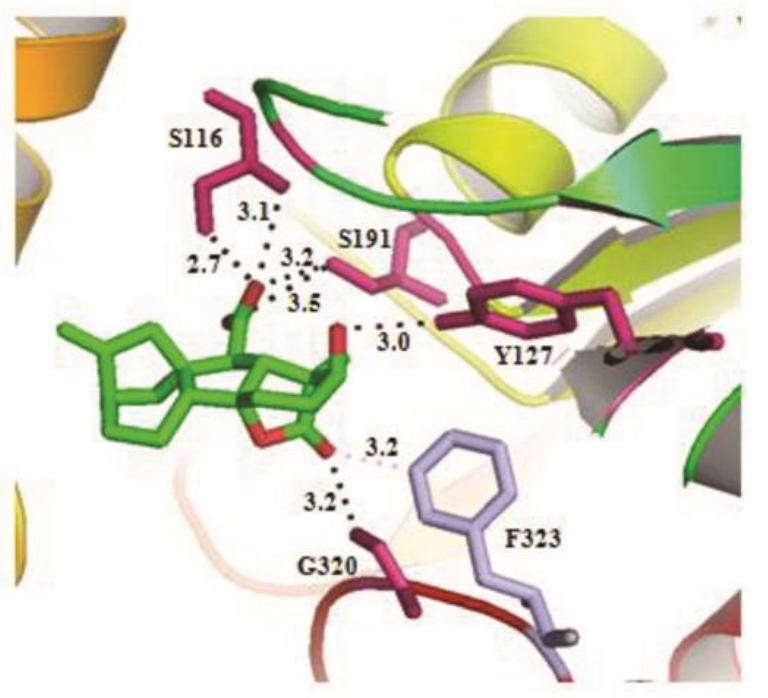
and; D) mutated structure of 2ZSH-GA4. Amino acid Leu ${ }^{323}$ of GID1a and its corresponding amino acid in GID1b (Phe ${ }^{323}$ ) are shown in light blue; distances of these amino acids to $\mathrm{GA}_{3} / \mathrm{GA}_{4}$ are also shown in dotted light blue. Hydrogen bonds are shown in dotted black lines. 